\title{
Susceptibilities of epicardial and subcutaneous fat tissue for browning-gene expression and diet-induced volume reduction are different
}

\author{
HIROSHI MIKAMO ${ }^{1}$, MEIZI JIANG ${ }^{2}$, MAHITO NORO $^{1}$, YASUO SUZUKI $^{3}$, NOBUYUKI HIRUTA $^{4}$, \\ HIROYUKI UNOKI-KUBOTA ${ }^{5}$, WOLFGANG J. SCHNEIDER ${ }^{6}$ and HIDEAKI BUJO ${ }^{2}$
}

\begin{abstract}
Departments of ${ }^{1}$ Cardiology, ${ }^{2}$ Clinical-Laboratory and Experimental-Research Medicine, ${ }^{3}$ Internal Medicine and ${ }^{4}$ Pathology, Toho University Sakura Medical Center, Sakura, Chiba 285-8741; ${ }^{5}$ Department of Diabetic Complications, Diabetes Research Center, Research Institute, National Center for Global Health and Medicine, Tokyo 162-8655, Japan; ${ }^{6}$ Department of Medical Biochemistry, Max F. Perutz Laboratories, Medical University of Vienna, Vienna 1090, Austria
\end{abstract}

Received November 8, 2017; Accepted January 25, 2018

DOI: $10.3892 / \mathrm{mmr} .2018 .8690$

\begin{abstract}
The upregulation of brown or brown-like beige adipocytes is a potential strategy for the prevention or treatment of diabetes and coronary artery diseases in obese patients. Epicardial adipose tissue (EAT) differs significantly from subcutaneous fat tissue (SAT) in metabolic properties. To investigate properties of EAT further, thermogenesis gene expression was investigated in human autopsy and murine samples, and adipocytes differentiated from EAT mesenchymal cells. Subsequently, analyzed EAT volume alterations were observed to be associated with weight reduction in obese patients by imaging. Gene expression analyses of autopsy samples revealed that UCP-1 mRNA levels in EAT were significantly increased compared with SAT, and $\beta 3$-adrenergic receptor (AR) levels tended to be increased; this finding was verified in comparing EAT with SAT in mice. Browning stimulation of human EAT-derived MCs increased uncoupling protein-1 and $\beta 3$-AR levels by 3.2 fold- and 12.6-fold compared with SAT-derived MCs, respectively. Subsequent imaging for EAT volume measurement using multi-detector computed tomography in 10 obese patients revealed that mean EAT volumes did not significantly decrease following weight loss therapy. The EAT volume alterations were not correlated with weight changes, whereas positive correlations were observed in SAT and visceral adipose tissue. Therefore, the studies in man and mouse on EAT properties demonstrated that susceptibilities of EAT and SAT for browning-gene
\end{abstract}

Correspondence to: Professor Hideaki Bujo, Department of Clinical-Laboratory and Experimental-Research Medicine, Toho University Sakura Medical Center, 564-1 Shimoshizu, Sakura, Chiba 285-8741, Japan

E-mail: hideaki.bujo@med.toho-u.ac.jp

Key words: EAT, weight reduction, autopsy, thermogenesis, gene expression expression and diet-induced volume reduction were grossly different. The data suggest a potential association of EAT with local thermogenetic and metabolic homeostasis in cardiac and/or cardiovascular cells, in conjunction with systemic energy metabolism.

\section{Introduction}

Animal studies have revealed that increasing heat production in adipose tissue can offset obesogenic effects of a high-fat diet, whereas reducing thermogenesis exacerbates them (1). The existence of brown adipose tissue (BAT) in adult humans has been reconfirmed by using modern imaging techniques and tissue biopsies $(2,3)$, increasing the importance of understanding the pathophysiological significance of brown and brown-like 'beige' adipocytes as players for systemic metabolism and also neighboring tissue thermo-regulation. In fact, the levels of detectable BAT negatively correlate with age, body mass index (BMI) and diabetes conditions (4). Thus, an increasing number of factors and molecules have been identified to increase the numbers or thermogenic activities of brown/beige adipocytes. In this context, we recently identified a key thermogenesis inhibitor that functions in response to changing environmental conditions and particularly when thermogenic activity is enhanced in capable tissues as a fine-tuning negative regulator (5).

Epicardial adipose tissue (EAT) is a cluster of metabolically and thermogenetically unique fat cells among various fat tissues (6). Accumulated evidence for the gene expression patterns in human and animal tissues have strengthened the biochemical findings $(7,8)$ that EAT is characterized by increased lipolytic property with $\beta$-adrenergic activities $(9,10)$. Recent histochemical and cell-biological studies indeed have demonstrated that human adult epicardial adipocytes share features with the above-mentioned brown/beige adipocytes (11). Thus, how the unique character is associated with cardiac thermo-stasis and systemic metabolism is an issue to be solved, in order to exploit the regulation of beige/brown adipocytes towards 
prevention and treatment of diabetes and coronary artery diseases (CAD) in obese patients.

In the present study, we first investigated the expression of genes involved in thermogenesis in human EAT, in comparison to subcutaneous adipose tissue (SAT), from 15 autopsy samples and also in mouse EAT. Furthermore, the sensitivities of the gene expression in human mesenchymal cells (MCs)-derived brown adipocytes prepared from EAT and SAT samples were elucidated. Third, we evaluated the susceptibilities of EAT volume changes against weight reduction using multi-detector computed tomography (MDCT) in 10 obese patients in comparison to those of SAT and visceral adipose tissue (VAT).

\section{Materials and methods}

Autopsy samples. The study protocol for the collection and analyses of autopsy samples was approved by the Ethics Committee of Toho University Sakura Medical Center, in accordance with the Declaration of Helsinki. Each family gave informed consent before the study began for the study use of autopsy subject sample. Eighteen consecutive patients who underwent autopsy were originally recruited in this study (Table I), and among them, two cases were excluded because of treatment histories with thyrotoxic crisis (sample no. 18), and intensive therapy with long-time noradrenergic stimulation for acute myocardial infarction (sample no. 3), respectively. As a result, sixteen autopsy samples were included in this study. Samples of EAT and SAT were obtained from the distal left anterior descending artery at the apex of the heart, and upper abdominal wall, respectively. Fat samples were trimmed of connective tissue and superficial blood vessels, and stored at $-80^{\circ} \mathrm{C}$ for the subsequent gene expression analysis. The samples from 15 cases (sample nos. 1, 2, 4, 5, 6, 7, 8, 9, 10, 11, 12, 13, 15, 16, and 17) were used for gene expression analysis, and the sample no. 14 was used for cell culture experiments without gene expression analysis, because of the volume limitation of prepared fat.

Mouse experiments. All data were from work on male (56 weeks of age) $d \mathrm{~b} / d \mathrm{~b}$ mice, which were purchased from Clea (Tokyo, Japan), and housed in a temperature-controlled room $\left(22^{\circ} \mathrm{C}\right)$ with a 12 -h light/dark cycle with free access to food and water. Standard chow was administered ad libitum from weaning. EAT and SAT were obtained as described in 2.1. autopsy samples, and stored at $-80^{\circ} \mathrm{C}$ for the subsequent gene expression analysis. All animal procedures were approved by the local animal ethics committee of the National Center for Global Health and Medicine.

Cell culture. Experiments were performed with the adipose tissue specimens obtained from an autopsy sample (sample no. 14; Table I). Human primary MCs were prepared from the stromal vascular fraction of fat tissues, and cultured as described by excision of the fat pad followed by mincing with sterile surgical scissors and digestion at $37^{\circ} \mathrm{C}$ with shaking for $30 \mathrm{~min}$ (5). Digests were filtered and left to sit on ice for $10 \mathrm{~min}$. The upper fat layer was removed and then the upper $2 / 3$ of the supernatant washed twice in PBS (Wako) before being plated. Cells were maintained at $37^{\circ} \mathrm{C}$ in $5 \% \mathrm{CO}_{2}$ in Dulbecco's modified Eagle's medium (Wako) with $10 \%$ fetal bovine serum (Gibco; Thermo Fisher Scientific, Tokyo, Japan),
$20 \mathrm{mM}$ L-glutamine (Wako), 100 units/ml, penicillin, and $100 \mu \mathrm{g} / \mathrm{ml}$ streptomycin (both from Gibco; Thermo Fisher Scientific). The cells were further incubated in the medium of the above composition with/without $0.25 \mu \mathrm{M}$ dexamethasone (Wako), $0.5 \mathrm{mM}$ 3-isobutyl-1-methylxanthine (IBMX; Biovision, Milpitas, CA, USA), $125 \mu \mathrm{M}$ indomethacin, $850 \mathrm{~nm}$ insulin (Wako), and $1 \mathrm{nM} \mathrm{3,} \mathrm{30,} \mathrm{5-Triiodo-thyronine}\left(\mathrm{T}_{3}\right.$; Sigma-Aldrich, Tokyo, Japan) for 2 days, accompanied by subsequent incubation with $850 \mathrm{nM}$ insulin and $1 \mathrm{nM} \mathrm{T}$ for 5 days for browning differentiation. After 7 days, cells were fully differentiated (validated by morphological appearance) and used for gene expression experiments. The procedures were approved by the Ethical Committee on human study, Toho University Sakura Medical Center.

Reverse transcription-quantitative polymerase chain reaction (RT-qPCR).RT-qPCR experiments were performed as described previously (12). Total RNA was prepared from tissues or cultured cells using the Maxwell ${ }^{\circledR} 16$ LEV simply RNA Purification kit, and immediately quantified using the Quantus ${ }^{\mathrm{TM}}$ Fluorometer (both Promega, Tokyo, Japan). For cDNA synthesis, the reverse transcription reaction was performed with the Affinity Script QPCR cDNA Synthesis kit (Agilent Technologies Japan, Tokyo, Japan). The target fragments of the cDNA samples were amplified by Applied Biosystems ${ }^{\circledR}$ StepOnePlus $^{\mathrm{TM}}$ using TaqMan Gene Expression Assay with TaqMan Fast Advanced Master Mix (both Applied Biosystems; Thermo Fisher, Yokohama, Japan) with the combinations of primers and probes for UCP1 (Hs00222453_m1 or Mm01244861_ $\mathrm{m} 1), \beta 3$-adrenergic receptor (AR) (Hs00609046_m1 or Mm02601819_g1), leptin (Hs00174877_m1 and Mm00434759_ m1), TNF- $\alpha$ (Hs00174128_m1 or Mm00443258_ml), VEGF (Hs00900055_m1 or Mm00437304_m1) or 18S RNA (Hs99999901_s1). For the experiments using MCs, samples obtained from cells after browning stimulation treatment were used as calibrator to allow comparison of relative mRNA levels in the assays.

Human study. We prospectively enrolled ten consecutive obese patients in this study, who were admitted to the Department of Cardiology, Toho University Sakura Medical Center (Sakura, Japan) due to stable angina or heart failure, and underwent a regular weight reduction program using low-calorie diet and aerobic exercise (Table II). Patients with malignant diseases, inflammatory diseases, or under hemodialysis, were excluded from the study. Diabetes mellitus was defined by either a value $>6.5 \%$ of glycosylated hemoglobin (HbA1c) (13) or being under medication with oral hypoglycemic drugs. Among the study subjects, there were none on insulin therapy, five on oral hypoglycemic agents, and one on dietary therapy alone. Patients with systolic blood pressure (SBP) $\geqq 140 \mathrm{mmHg}$ or diastolic blood pressure (DBP) $\geqq 90 \mathrm{mmHg}$ (14), or under anti-hypertensive medication were considered hypertensive. Dyslipidemia was defined as low-density lipoprotein cholesterol (LDL-C) $\geqq 140 \mathrm{mg} / \mathrm{dl}$, high-density lipoprotein cholesterol (HDL-C) $<40 \mathrm{mg} / \mathrm{dl}$, or triglyceride $\geqq 150 \mathrm{mg} / \mathrm{dl}$ (15), or being under treatment with statins and/or lipid-lowering agents. We defined renal dysfunction as an estimated glomerular filtration rate (eGFR) of $<60 \mathrm{ml} / \mathrm{min} / 1.73 \mathrm{~m}^{2}(16)$, and calculated the eGFR using baseline serum creatinine. Eight patients were 


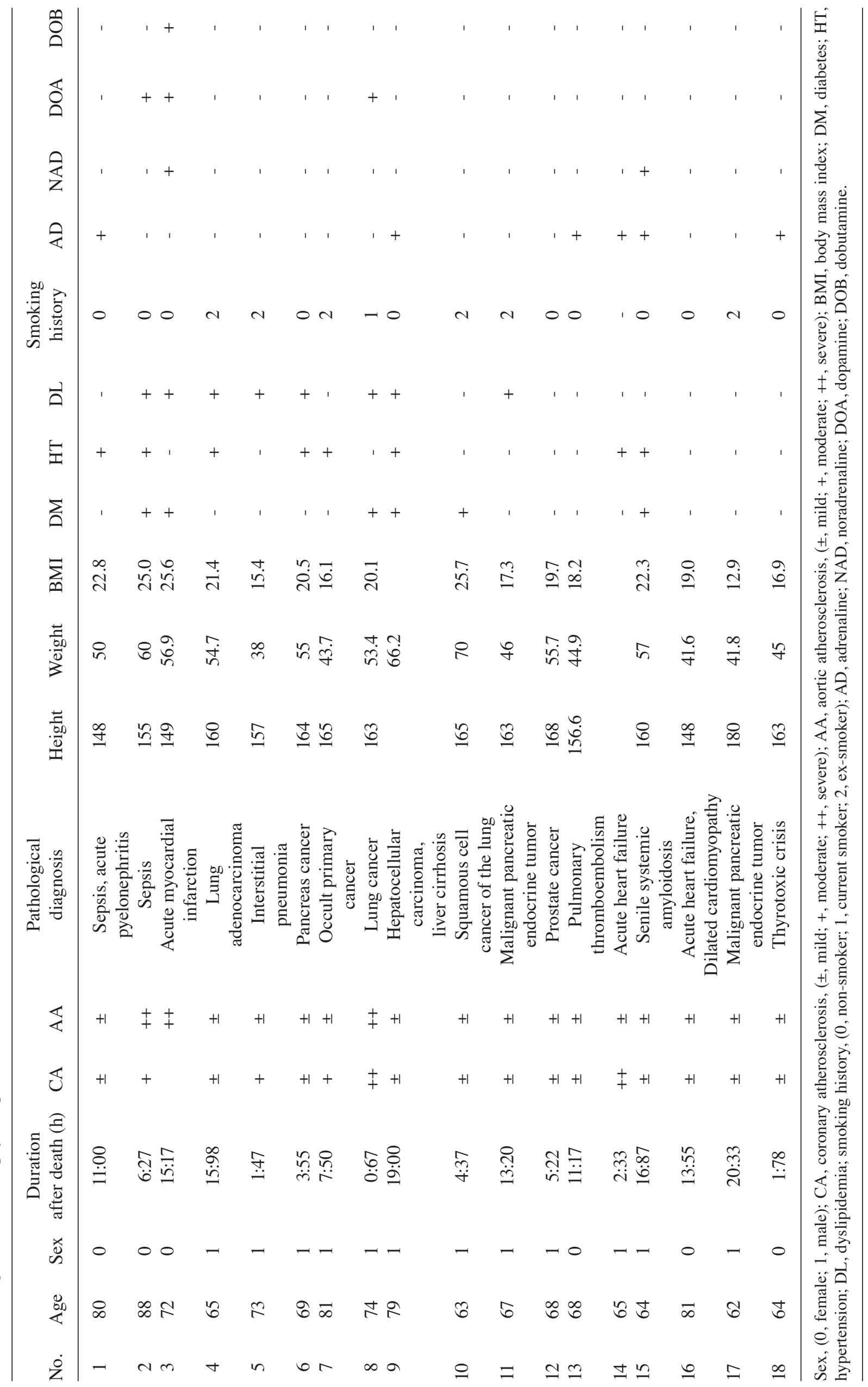


Table II. Characteristics and biochemistry of study subjects before and after weight reduction therapy.

\begin{tabular}{|c|c|c|c|c|}
\hline Variables & Before & & After & P-value \\
\hline Age (years) & & $44.6 \pm 16.2$ & & - \\
\hline Male & & $8(80 \%)$ & & - \\
\hline Weight loss duration (days) & & $44.1 \pm 27.6$ & & - \\
\hline Current smoker & & $5(50 \%)$ & & - \\
\hline $\mathrm{TC}(\mathrm{mg} / \mathrm{dl})$ & $177.3 \pm 45.8$ & & $160.5 \pm 32.1$ & 0.235 \\
\hline TG (mg/dl) & $118.1 \pm 40.1$ & & $105.4 \pm 23.1$ & 0.445 \\
\hline HDL-C (mg/dl) & $38.2 \pm 5.6$ & & $39.2 \pm 7.5$ & 0.735 \\
\hline LDL-C (mg/dl) & $117.5 \pm 43.4$ & & $99.6 \pm 32.0$ & 0.097 \\
\hline $\mathrm{SBP}(\mathrm{mmHg})$ & $136.0 \pm 19.8$ & & $129.7 \pm 17.7$ & 0.144 \\
\hline $\mathrm{DBP}(\mathrm{mmHg})$ & $80.7 \pm 13.1$ & & $75.7 \pm 10.6$ & 0.216 \\
\hline Heart rate (beats/min) & $72.6 \pm 11.8$ & & $65.7 \pm 7.8$ & 0.085 \\
\hline FPG (mg/dl) & $112.5 \pm 35.4$ & & $98.4 \pm 18.4$ & 0.234 \\
\hline HbA1c (JDS) (\%) & $6.9 \pm 1.3$ & & $6.2 \pm 0.7$ & 0.084 \\
\hline Creatinine (mg/dl) & $1.08 \pm 0.4$ & & $1.94 \pm 2.6$ & 0.324 \\
\hline Uric acid (mg/dl) & $7.6 \pm 2.4$ & & $6.5 \pm 1.5$ & 0.100 \\
\hline \multicolumn{5}{|l|}{ Complications } \\
\hline CAD & & $2(20 \%)$ & & \\
\hline Renal dysfunction & & $3(30 \%)$ & & \\
\hline Medications & & $5(50 \%)$ & & \\
\hline \multicolumn{5}{|l|}{ Diabetes excluding insulin } \\
\hline Insulin supplementation & & $0(0 \%)$ & & \\
\hline Hypertension & & $9(90 \%)$ & & \\
\hline Dyslipidemia & & $3(30 \%)$ & & \\
\hline
\end{tabular}

Values are mean $\pm \mathrm{SD}$ or $\mathrm{n}(\%)$. The statistical differences were analyzed by the paired Student $\mathrm{t}$-test. TC, total cholesterol; TG, triglyceride; HDL-C, high-density lipoprotein cholesterol; LDL-C, low-density lipoprotein cholesterol; SBP, systolic blood pressure; DBP, diastolic blood pressure; FPG, fasting plasma glucose; HbA1c, hemoglobin A1c; JDS, Japan Diabetes Society; CAD, coronary artery disease.

treated with angiotensin receptor blockers, and three patients received statins. Smoking habits and the amount of tobacco consumed were examined in the questionnaire and subjects were classified into three categories; non-smokers (those who have not smoked more than 100 cigarettes in total or more than 6 months so far), current smokers (those who have smoked more than 100 cigarettes in total or more than 6 months so far and who smoke daily or occasionally in the past month), or ex-smokers (those who have smoked more than 100 cigarettes in total or more than 6 months so far and who have not smoked in the past month). The diagnosis of CAD was based on a history of myocardial infarction or angina pectoris. Obesity was defined as BMI $\geq 25 \mathrm{~kg} / \mathrm{m}^{2}(17)$. The subjects had low calorie diet with partial replacement of meal by Formula diet (Microdiet ${ }^{\circledR}$; Sunny Health, Tokyo, Japan) once a day at dinner (974 kcal/day) for 3 weeks, and subsequently without the replacement $(1,200 \mathrm{kcal} /$ day $)$ until discharge. The exercise therapy was continued for $20 \mathrm{~min}$ using an ergometer or a treadmill based on the Borg scale. The body weights and lengths before and after the program achievement for the periods ranging 3 weeks to 2 months were measured together with other biochemical parameters. Throughout the weight reduction program, the patients' compliance with mandated calorie intake and aerobic exercise was monitored. The study protocol was approved by the Ethics Committee of Toho University Sakura Medical Center. All subjects gave informed consent before the study began.

CT measurement. The volume of EAT with areas of SAT and VAT were measured before and after the performance of the above weight reduction program using MDCT. All scans were performed using a 64-slice CT scanner (Aquillion $64^{\circledR}$; Toshiba, Tokyo, Japan). A non-enhanced CT was performed by retrospective ECG gating according to the following protocol; a tube voltage of $120 \mathrm{kV}$ and tube current $200 \mathrm{~mA}$ using ECG-correlated tube current modulation. EAT volume was quantified by the sum of areas of EAT on cross-sectional CT images from the root of coronary arteries to the apex using the volume analysis software tool of a workstation (18). In detail, the axial source image was traced on the pericardial sac manually from the left main trunk to the left ventricular apex. The 3-dimentional image of the heart was constructed by totaling of these slices. Fat voxels were identified using the threshold attenuation values of -150 to $-50 \mathrm{HU}$. The total volume was EAT as a result of the processing. The areas of SAT and VAT were determined by measuring a -150 to $-50 \mathrm{HU}$ areas using a modification of the method of CT scanning at the cross-sectional umbilical level (19). 

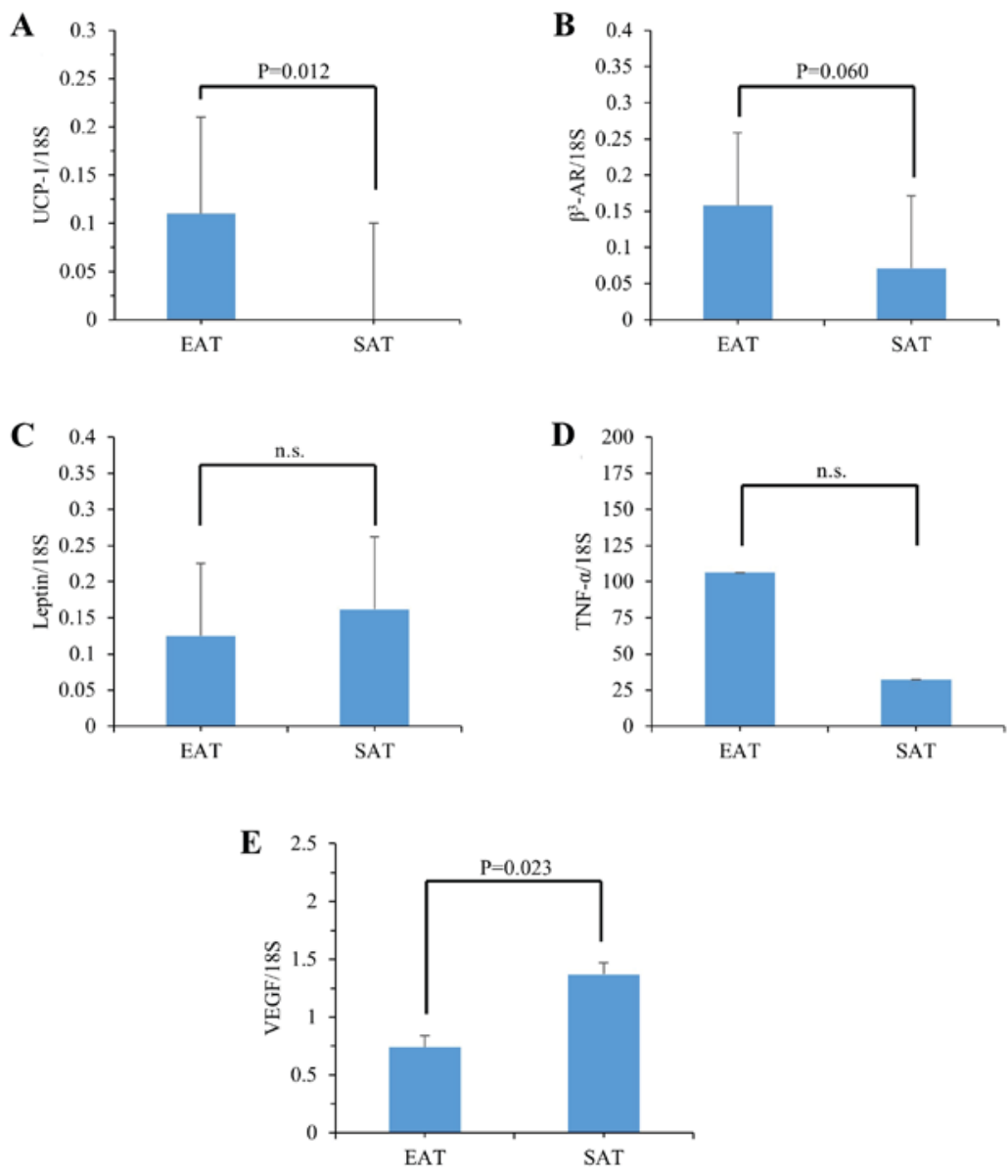

Figure 1. The levels of UCP-1 (A) $\beta 3-A R$ (B) leptin (C) TNF- $\alpha$ (D) and VEGF (E) in EAT or SAT from human autopsy samples. Each mRNA level was analyzed by reverse transcription-polymerase chain reaction, as described in Materials and Methods. The mRNA levels relative to 18S RNA levels were calculated, and expressed as the mean \pm standard deviation $(n=15)$. The statistical differences were analyzed by Mann-Whitney U-test. n.s., not significant; UCP, uncoupling protein; EAT, epicardial adipose tissue; SAT, subcutaneous adipose tissue; $\beta 3$-AR, $\beta 3$-adrenergic receptor.

Statistical analysis. Data in the text, tables, and figures are expressed as mean \pm SD For gene expression analysis, differences were analyzed by unpaired Student t-test or Mann-Whitney U-test. For human imaging study, differences in continuous variables were analyzed by unpaired Student t-test. A t-test with 95\% confidence interval (CI) was used to calculate differences of EAT volume, VAT and SAT area, and weight, between baseline and after weight reduction. Reductions (\%) in study parameters were presented by the proportions (\%) of reduced values at the endpoints of program from the values before starting the program. Relations between the reductions among weights, EAT, VAT, and SAT were analyzed using correlation analysis with linear-regression analyses. These results are presented as coefficient of determination $\left(\mathrm{r}^{2}\right)$, which indicates the percentage of variation in the dependent variable that can be explained by the independent variables, with the ranges of 95\% CI. Statistical analyses were performed using PASW Statistics 18 (SPSS Inc., Chicago, IL, USA), and P $<0.05$ was considered to indicate a statisticaly significant difference.

\section{Results}

Thermogenesis genes are increased in human autopsy EAT. In order to determine the expression of genes involved in the thermogenetic function of EAT, we first studied two genes essential for the thermogenic activity in brown/beige adipocytes, i.e., uncoupling protein (UCP)-1 and $\beta 3$-AR, and the expression of leptin, TNF- $\alpha$ and VEGF as representative genes involved in adipogenesis, inflammation, and angiogenesis, respectively, in 15 autopsy EAT and SAT samples (Fig. 1; Table I). The expression levels of UCP-1 were significantly increased in EAT (Fig. 1A), and those of $\beta 3$-AR tended to be increased in EAT (Fig. 1B), compared with those of SAT, respectively. There were no significant differences between EAT and SAT in the expression of leptin (Fig. 1C) or TNF- $\alpha$ (Fig. 1D), and the expression of VEGF was significantly decreased in EAT, compared with that in SAT (Fig. 1E). These results suggested that the expression of genes essential for thermogenesis in brown/beige adipocytes was specifically induced in EAT relative to SAT.

Thermogenesis genes are reproducibly induced in mouse $E A T$. Because the heterogeneous pathological backgrounds in human autopsy samples may cause the increased expression of thermogenesis genes, we next analyzed the expression of the same genes in a mouse model, i.e., $d \mathrm{~b} / d \mathrm{~b}$ mice (Fig. 2), because considerable amounts of EAT were observed under diabetic conditions in these animals (data not shown). Significantly, 

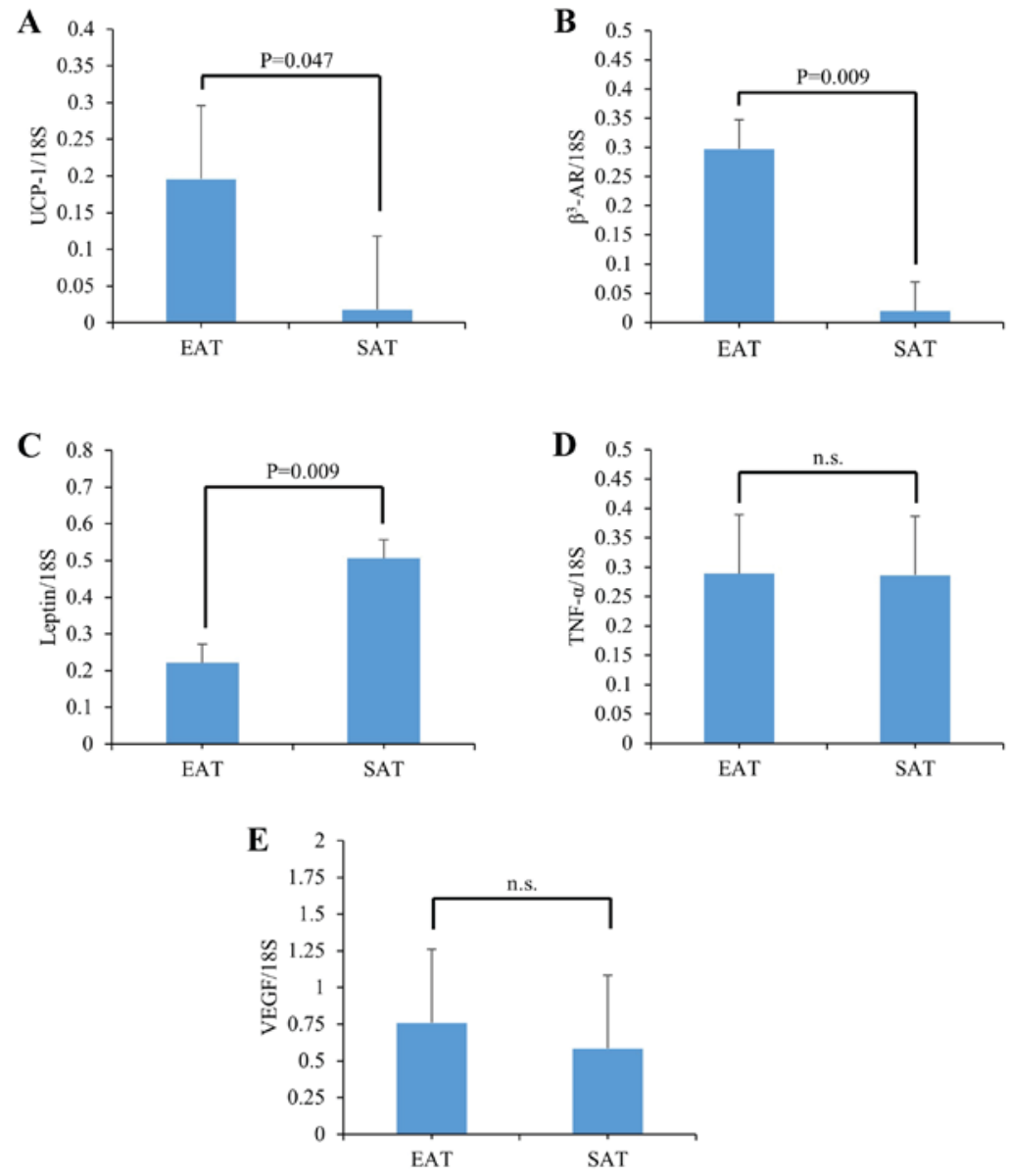

Figure 2. The levels of UCP-1 (A) $\beta 3$-AR (B) leptin (C) TNF- $\alpha$ (D) and VEGF (E) in EAT or SAT from a mouse model ( $d \mathrm{~b} / d \mathrm{~b})$. Each mRNA level was analyzed by reverse transcription-polymerase chain reaction as described in Materials and Methods. The mRNA levels relative to 18S RNA levels were calculated and expressed as the mean \pm standard deviation $(\mathrm{n}=5)$. The statistical differences were analyzed by Mann-Whitney U-test. n.s., not significant; UCP, uncoupling protein; EAT, epicardial adipose tissue; SAT, subcutaneous adipose tissue; $\beta 3$-AR, $\beta 3$-adrenergic receptor.
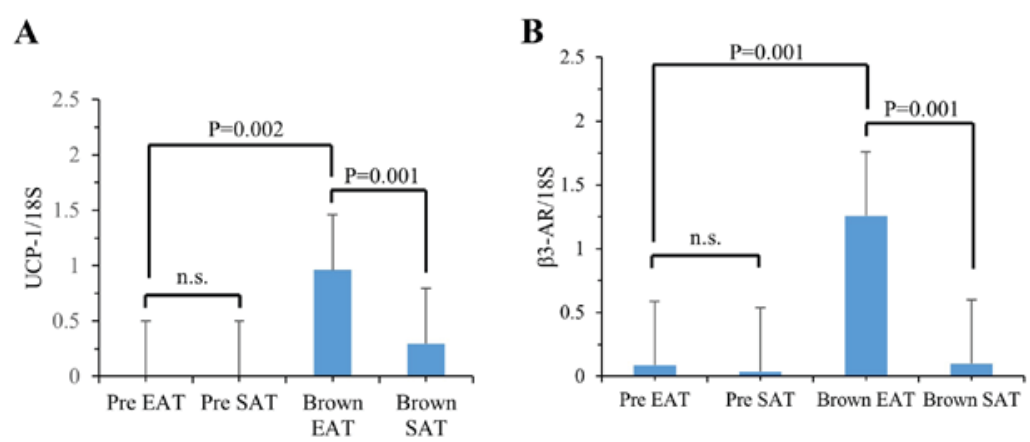

Figure 3. The levels of mRNA for UCP-1 (A) and $33-\mathrm{AR}$ (B) in MCs from EAT or SAT of a human autopsy sample. mRNA was prepared from the cells incubated with/without browning differentiation stimulation for 8 days, and each mRNA level was analyzed by reverse transcription-polymerase chain reaction, as described in Materials and Methods. mRNA levels relative to 18S RNA levels were calculated and expressed as the mean \pm standard deviation ( $\mathrm{n}=3$ ). The statistical differences were analyzed by the unpaired Student t-test. Pre EAT, MCs from EAT without differentiation stimulation; pre SAT, MCs from SAT without differentiation stimulation; brown EAT, MCs from EAT with browning differentiation stimulation; brown SAT, MCs from SAT with browning differentiation stimulation. n.s., not significant; UCP, uncoupling protein; MCs, mesenchymal cells; EAT, epicardial adipose tissue; SAT, subcutaneous adipose tissue; $\beta 3$-AR, $\beta 3$-adrenergic receptor.

the levels of UCP-1 and $\beta 3$-AR were increased 16.6-fold and 15.2-fold over those in SAT, respectively (Fig. 2A and B). On the other hand, leptin expression levels were significantly decreased in EAT compared with those in SAT ((Fig. 2C). The TNF- $\alpha$ and VEGF levels were not different between EAT and SAT ((Fig. 2D and E). Thus, the increases of thermogenesis genes UCP- 1 and $\beta 3$-AR, observed in the human autopsy EAT of various pathological backgrounds, were reproduced in an analogous mouse model.

Expression of thermogenesis genes is drastically induced in response to differentiation stimulation in MCs from 
Table III. Adipose tissues and weights of obese patients before and after weight reduction therapy and their reductions after therapy.

\begin{tabular}{lccr}
\hline Variables & Before $(\mathrm{n}=10)$ & After $(\mathrm{n}=10)$ & P-value \\
\hline EAT $(\mathrm{ml})$ & $248.65 \pm 36.57$ & $237.47 \pm 57.17$ & 0.249 \\
VAT $\left(\mathrm{mm}^{2}\right)$ & $190.93 \pm 74.78$ & $153.91 \pm 72.70$ & 0.001 \\
SAT $\left(\mathrm{mm}^{2}\right)$ & $431.63 \pm 206.76$ & $375.37 \pm 206.16$ & 0.003 \\
Body weight $(\mathrm{kg})$ & $109.54 \pm 28.87$ & $100.24 \pm 26.73$ & $<0.001$ \\
BMI $\left(\mathrm{kg} / \mathrm{m}^{2}\right)$ & $38.75 \pm 9.36$ & $35.45 \pm 8.65$ & $<0.001$ \\
Reduction in weight $(\%)$ & & & \\
Reduction in BMI (\%) & & $8.43 \pm 3.19$ & \\
Reduction in EAT $(\%)$ & $5.49 \pm 3.19$ & \\
Reduction in VAT (\%) & & & \\
Reduction in SAT $(\%)$ & & $20.61 \pm 14.77$ & \\
\end{tabular}

Values are mean \pm SD The statistical differences were analyzed by the paired Student t-test. EAT, epicardial adipose tissue; VAT, visceral adipose tissue; SAT, subcutaneous adipose tissue; BMI, body mass index; reductions, reductions from baseline (\%) which were calculated by the equation of (values before therapy - values after therapy)/(values before therapy) x 100 .

human autopsy EAT. In order to reveal the mechanism by which the expression levels of thermogenesis genes were increased in the human autopsy and murine EAT samples, we studied the regulation of gene expression in response to the stimulation of differentiation to brown mature adipocytes in MCs prepared from human autopsy EAT and SAT samples (Fig. 3). The UCP-1 expression levels were drastically increased in MCs both from human EAT and SAT after differentiation stimulation when compared with those in undifferentiated MCs (Fig. 3A). The expression levels of UCP-1 in differentiated adipocytes from EAT were 3.2-fold higher than those in SAT, whereas the levels in undifferentiated MCs were not significantly different between EAT and SAT. The $\beta 3$-AR expression levels were also increased in differentiated MCs, compared with those in undifferentiated MCs, both from EAT and SAT (Fig. 3B). Finally, the 33 -AR levels in EAT were 12.6-fold higher than those in SAT, whereas the levels in undifferentiated MCs from EAT or SAT were not significantly different. These results indicate that the sensitivity change in response to the differentiation stimulations to mature brown adipocytes in MCs was much more pronounced in EAT than that in SAT, and thus, the different sensitivity of MCs may cause the differences in thermogenesis gene expression between EAT and SAT in humans.

The sensitivity of the reduction in accumulated EAT against weight reduction therapy is different from those in SAT in obese patients. In order to define the pathophysiological properties of human EAT in the clinical setting and considering the above characterized differential features of cultured cells, we performed a comparative MDCT imaging study in obese patients on a weight reduction program for the evaluation of changes in the volumes of EAT, and by CT in SAT and VAT. The 10 patients' (8 males and 2 females; $44.6 \pm 16.2$ years old) body weights at the beginning and at the end of the weight reduction (duration of therapy, 44.1 \pm 27.6 days) were $109.54 \pm 28.87 \mathrm{~kg}$ and $100.24 \pm 26.73 \mathrm{~kg}$, respectively (Tables II and III). After completion of the therapy, in addition to body weight, values of SAT and VAT were also significantly decreased in the patients when compared to the values before therapy. However, the degrees of EAT were not significantly different before and after the therapy. The proportions of reductions in weights were clearly and positively correlated with those in SAT $\left(\mathrm{r}^{2}=0.487\right.$, $\mathrm{P}=0.025)$ and $\operatorname{VAT}\left(\mathrm{r}^{2}=0.806, \mathrm{P}<0.001\right)$, but not with those in EAT $\left(r^{2}=0.103, P=0.365\right)$ (Table IV). The reductions in EAT were not correlated with those in SAT $\left(r^{2}=0.259, P=0.133\right)$, nor with those in VAT $\left(r^{2}=0.158, P=0.256\right)$, whereas there was a clear positive correlation between SAT and VAT $\left(r^{2}=0.614\right.$, $\mathrm{P}=0.007$ ) (Table V). These results, obtained from a multiple imaging study using CT, suggest that in obese patients the sensitivity of the accumulated EAT against weight reduction therapy by dietary calorie restriction with daily exercise is different from those in SAT and VAT.

\section{Discussion}

In the present study, we showed that the expression of thermogenesis genes is induced in human EAT, in comparison to those in SAT, in 15 autopsy samples, and that the specific changes in expression pattern was also observed in murine EAT. Furthermore, the enhanced expression in EAT relative to that in SAT was also clearly present in the human MCs prepared from an autopsy EAT sample after differentiation stimulation to brown adipocytes. Thus, human tissue and cell analyses revealed an increased expression of thermogenesis genes as a unique property of EAT. A subsequent CT-imaging study demonstrated that the volume changes in the EAT accumulation by weight reduction therapy was not related to the weight reduction itself, whereas those of SAT and VAT were clearly associated with the weight reduction in obese patients. These results obtained in tissues and cells, and by imaging, indicate that EAT is developmentally rich in brown/beige adipocytes in comparison to SAT, and suggest that these cellular characteristics may be involved in the 
Table IV. Comparisons of reductions in adipose tissues with weights after therapy.

\begin{tabular}{lcrr}
\hline Variables & $\mathrm{r}^{2}$ & \multicolumn{1}{c}{$95 \%$ CI } & P-value \\
\hline Reduction in EAT & 0.103 & $-0.112 \sim 0.271$ & 0.365 \\
Reduction in VAT & 0.806 & $0.116 \sim 0.272$ & $<0.001$ \\
Reduction in SAT & 0.487 & $0.032 \sim 0.356$ & 0.025 \\
\hline
\end{tabular}

Comparisons of reductions in adipose tissues with weights were performed using correlation analysis with linear regression analysis. $r^{2}$, coefficient of determination; CI, confidence interval; EAT, epicardial adipose tissue; VAT, visceral adipose tissue; SAT, subcutaneous adipose tissue; reductions, reductions from baseline (\%) which were calculated by the equation of (values before therapy-values after therapy)/(values before therapy) x100.

unique sensitivity of EAT against weight reduction therapy, significantly different from other adipose tissues.

We have recently identified a key thermogenesis inhibitor, sLR11, which functions in maintaining the adequate balance between lipid storage and oxidation in response to changing environmental conditions (5). The deletion of LR11 in mice causes the ectopic appearance of beige/brown adipocytes in subcutaneous white adipose tissues associated with significantly enhanced expression levels of UCP-1 and $\beta 3-A R$. These changes were accompanied by a phenotype of increased cellular, and also systemic, energy expenditure in response to adrenergic stimulations in the ectopic beige/brown adipocytes (5). EAT has been reported to consist of a cluster of unique adipocytes among various fat tissues, the metabolic characteristics of which are increased lipolysis properties and $\beta$-adrenergic activities $(7,8)$. Accumulating evidence using gene expression analyses of human tissues and animal models have shown enhanced expression of UCP- 1 and $\beta 3$-AR in EAT, suggesting an increased activity of thermogenesis together with lipolysis in response to adrenergic stimulation $(9,10)$. In this study, we have shown that the enhanced expression of UCP-1 and $\beta 3$-AR in human-autopsy and mouse EAT were manifest in MCs derived from a human autopsy EAT sample after stimulation of differentiation to brown adipocytes; notably, the enhancement after stimulation was clearly more evident in MCs derived from EAT than from SAT. These results from cell culture experiments may indicate a thermogenetic character in human EAT different from that of other fat tissues, at least from SAT. The EAT characteristics likely are based on the differences in the properties and/or numbers of precursor cells in the tissue, rather than on the cells' environmental conditions. Thus, the necessity for expanding these studies into analyses using other clones from human epicardial MCs is clearly indicated.

Another (patho-)physiological property which has been suggested by prior studies is the possibly different sensitivity of accumulated EAT to the weight reduction therapy in obese patients. However, the previous imaging studies have shown rather heterogeneous results concerning the associations of reductions in EAT with those in body weights, SAT, or VAT (20-23). One possible reason for these diverging results has been suggested to be the different imaging methodologies
Table V. Correlations between pairs of reductions among EAT, SAT and VAT.

\begin{tabular}{lccc}
\hline Variables & $\mathrm{r}^{2}$ & $95 \% \mathrm{CI}$ & P-value \\
\hline EAT vs. VAT & 0.158 & $-0.306 \sim 0.997$ & 0.256 \\
EAT vs. SAT & 0.259 & $-0.216 \sim 1.356$ & 0.133 \\
VAT vs. SAT & 0.614 & $0.356 \sim 1.659$ & 0.007 \\
\hline
\end{tabular}

Comparisons of reductions among adipose tissues were performed using correlation analysis with linear regression analysis. $r^{2}$, coefficient of determination; CI, confidence interval; EAT, epicardial adipose tissue; VAT, visceral adipose tissue; SAT, subcutaneous adipose tissue; Reductions, Reductions from baseline (\%) which were calculated by the equation of (values before therapy-values after therapy)/(values before therapy) x 100 .

used for the measurement of EAT accumulations (20); previous studies have often quantitated EAT thickness before and after weight reduction therapy using echocardiography, and the volume using MDCT or magnetic resonance imaging (MRI) only at the beginning, and thus the associations of weight or other adipose tissue reduction with the EAT reduction have been proposed to be better determined using MDCT (20). Consequently, here we analyzed the volumes of EAT before and after weight reduction therapy using MDCT in 10 obese patients. The results showed that reductions in the accumulation of EAT were not associated with those in weight or in SAT, whereas there were clear relationships between pairs of weight, SAT, and VAT, as previously reported $(22,24,25)$. Thus, taken together, our results support the view that the response of EAT accumulation differs from that of those in SAT accumulation, possibly uncovering another unique EAT property in addition to the observed enhanced thermogenesis gene expression.

Limitations of the present study are that the 15 autopsy samples are principally of heterogeneous origin (Table I), although samples from cases treated with intensive adrenergic therapy were excluded (see Materials and Methods). Particularly, the MCs from EAT and SAT were successfully prepared only from an autopsy sample. In the imaging study, subjects were collected by a single institute, and thus the subject number for extensive analysis using multi-variable analyses was rather small. The methods for comparisons of factors might have to be re-evaluated, in addition to the evaluation using the reduction (\%) in a future study, as the comparisons were performed between the volume of EAT $(\mathrm{ml})$ and the areas $\left(\mathrm{mm}^{2}\right)$ of SAT and VAT. Clearly, further studies using subjects with different characteristics (e.g., sex, age, BMI, and possibly, therapy program) would be helpful for the evaluation of the pathological significance of EAT in weight reduction therapy.

In conclusion, the present human tissue, cell, and imaging studies about EAT properties showed that the susceptibilities of EAT for expression of browning-genes and diet- and exercise-induced volume reduction were different from those of SAT. Considering the insights gained from this study, EAT may be proposed to constitute a unique adipose tissue reflecting the pathological status of the metabolic homeostasis 
of cardiac and/or cardiovascular cells, in addition to the systemic metabolism as observed in SAT and VAT.

\section{Acknowledgements}

The present study was supported, in part, by Grants-in-aid for Scientific Research from the Japanese Ministry of Education, Culture, Sports, Science and Technology to Hideaki Bujo (grant no. 24390231 and 15K15198) and Meizi Jiang (grant no. 24790907$)$.

\section{Competing Interests}

The authors declare that they have no competing interests.

\section{References}

1. Bostrom P, Wu J, Jedrychowski MP, Korde A, Ye L, Lo JC, Rasbach KA, Boström EA, Choi JH, Long JZ, et al: A PGC1- $\alpha$-dependent myokine that drives brown-fat-like development of white fat and thermogenesis. Nature 481: 463-468, 2012.

2. Cypess AM, Lehman S, Williams G, Tal I, Rodman D Goldfine AB, Kuo FC, Palmer EL, Tseng YH, Doria A, et al: Identification and importance of brown adipose tissue in adult humans. N Engl J Med 360: 1509-1517, 2009.

3. Zingaretti MC, Crosta F, Vitali A, Guerrieri M, Frontini A, Cannon B, Nedergaard J and Cinti S: The presence of UCP1 demonstrates that metabolically active adipose tissue in the neck of adult humans truly represents brown adipose tissue. FASEB J 23: 3113-3120, 2009.

4. Ouellet V, Routhier-Labadie A, Bellemare W, Lakhal-Chaieb L, Turcotte E, Carpentier AC and Richard D: Outdoor temperature, age, sex, body mass index and diabetic status determine the prevalence, mass and glucose-uptake activity of 18F-FDG-detected BAT in humans. J Clin Endocrinol Metab 96: 192-199, 2011.

5. Whittle AJ, Jiang M, Peirce V, Relat J, Virtue S, Ebinuma H, Fukamachi I, Yamaguchi T, Takahashi M, Murano T, et al: Soluble LR11/SorLA represses thermogenesis in adipose tissue and correlates with BMI in humans. Nat Commun 6: 8951, 2015.

6. Iacobellis G, Corradi D and Sharma AM: Epicardial adipose tissue: Anatomic, biomolecular and clinical relationships with the heart. Nat Clin Pract Cardiovasc Med 2: 536-543, 2005.

7. Marchington JM, Mattacks CA and Pond CM: Adipose tissue in the mammalian heart and pericardium: Structure, foetal development and biochemical properties. Comp Biochem Physiol B 94: 225-232, 1989 .

8. Mattacks CA and Pond CM: Site-specific and sex differences in the rates of fatty acid/triacylglycerol substrate cycling in adipose, tissue and muscle of sedentary and exercised dwarf hamsters (Phodopus sungorus). Int J Obes 12: 585-597, 1988.

9. Iacobellis G: Local and systemic effects of the multifaceted epicardial adipose tissue depot. Nat Rev Endocrinol 11: 363-371, 2015

10. Chechi K and Richard D: Thermogenic potential and physiological relevance of human epicardial adipose tissue. Int J Obes Suppl 5 (Suppl 1): S28-S34, 2015.
11. Sacks HS, Fain JN, Bahouth SW, Ojha S, Frontini A, Budge H, Cinti $\mathrm{S}$ and Symonds ME: Adult epicardial fat exhibits beige features. J Clin Endocrinol Metab 98: E1448-E1455, 2013.

12. Terai K, Jiang M, Tokuyama W, Murano T, Takada N, Fujimura K, Ebinuma H, Kishimoto T, Hiruta N, Schneider WJ and Bujo H: Levels of soluble LR11/SorLA are highly increased in the bile of patients with biliary tract and pancreatic cancers. Clin Chim Acta 457: 130-136, 2016.

13. Committee of the Japan Diabetes Society on the Diagnostic Criteria of Diabetes Mellitus, Seino Y, Nanjo K, Tajima N, Kadowaki T, Kashiwagi A, Araki E, Ito C, Inagaki N, Iwamoto Y, et al: Report of the committee on the classification and diagnostic criteria of diabetes mellitus. J Diabetes Investig 1: 212-228, 2010

14. Shimamoto K, Ando K, Fujita T, Hasebe N, Higaki J, Horiuchi M, Imai Y, Imaizumi T, Ishimitsu T, Ito M, et al: The japanese society of hypertension guidelines for the management of hypertension (JSH 2014). Hypertens Res 37: 253-390, 2014.

15. Teramoto T, Sasaki J, Ishibashi S, Birou S, Daida H, Dohi S, Egusa G, Hiro T, Hirobe K, Iida M, et al: Diagnostic criteria for dyslipidemia. J Atheroscler Thromb 20: 655-660, 2013.

16. Imai E, Matsuo S, Makino H, Watanabe T, Akizawa T, Nitta K, Iimuro S, Ohashi Y and Hishida A; CKD-JAC Study Group: Chronic kidney disease japan cohort (CKD-JAC) study: Design and methods. Hypertens Res 31: 1101-1107, 2008.

17. Examination Committee of Criteria for 'Obesity Disease' in Japan; Japan Society for the Study of Obesity: New criteria for 'obesity disease' in Japan. Circ J 66: 987-992, 2002.

18. Sarin S, Wenger C, Marwaha A, Qureshi A, Go BD, Woomert CA, Clark K, Nassef LA and Shirani J: Clinical significance of epicardial fat measured using cardiac multislice computed tomography. Am J Cardiol 102: 767-771, 2008.

19. Borkan GA, Gerzof SG, Robbins AH, Hults DE, Silbert CK and Silbert JE: Assessment of abdominal fat content by computed tomography. Am J Clin Nutr 36: 172-177, 1982.

20. Sacks HS: Weight loss in obesity reduces epicardial fat thickness; so what? J Appl Physiol (1985) 106: 1-2, 2009.

21. Rabkin SW and Campbell H: Comparison of reducing epicardial fat by exercise, diet or bariatric surgery weight loss strategies: A systematic review and meta-analysis. Obes Rev 16: 406-415, 2015.

22. Foppa M, Pond KK, Jones DB, Schneider B, Kissinger KV and Manning WJ: Subcutaneous fat thickness, but not epicardial fat thickness, parallels weight reduction three months after bariatric surgery: A cardiac magnetic resonance study. Int J Cardiol 168: 4532-4533, 2013.

23. Willens HJ, Byers P, Chirinos JA, Labrador E, Hare JM and de Marchena E: Effects of weight loss after bariatric surgery on epicardial fat measured using echocardiography. Am J Cardiol 99: 1242-1245, 2007.

24. Doucet E, St-Pierre S, Almeras N, Alméras N, Imbeault P, Mauriège P, Pascot A, Després JP and Tremblay A: Reduction of visceral adipose tissue during weight loss. Eur J Clin Nutr 56: 297-304, 2002.

25. Stallone DD, Stunkard AJ, Wadden TA, Foster GD, Boorstein J and Arger P: Weight loss and body fat distribution: A feasibility study using computed tomography. Int J Obes 15: 775-780, 1991.

This work is licensed under a Creative Commons Attribution-NonCommercial-NoDerivatives 4.0 International (CC BY-NC-ND 4.0) License. 